\title{
The Effect of Conscript General Training on Sleep Quality of Soldiers
}

\author{
Mollahadi. M ${ }^{1}$ \\ *Salaree. $\mathrm{MM}^{2}$ \\ 1- Ph.D. in Nursing, Assistant \\ Professor, Exercise Physiology \\ Research Center. Life Style \\ Institute, Nursing Faculty, \\ Baqiyatallah University of \\ Medical Sciences, Tehran, Iran. \\ 2- ( ${ }^{*}$ Corresponding Author) \\ Ph.D. in Nursing, Assistant \\ Professor, Health Research \\ Center, life Style Institute, \\ Nursing Faculty, Baqiyatallah \\ University of Medical Sciences, \\ Tehran, Iran. \\ Email:mmsalaree@gmail.com
}

\begin{abstract}
Introduction: Military personnel and soldiers, are at greater risk of public health and sleep disorders than civilians because of special missions, occupational issues, excessive psychological pressure and strict military laws.
\end{abstract}

Objective: The purpose of this study was to investigate the effect of conscript general training on the sleep quality of soldiers.

Materials and Methods: The present study was quasi-experimental with pre and post-intervention. The study population consisted of all soldiers in a general training center of military service in 2016. A total of 520 soldiers were studied using nonprobability and convenience sampling. The study was conducted in two stages before and after the completion of the military training course during about two months. Demographic and Pittsburgh Sleep Quality Index (PSQI) questionnaires were used for data collection. Finally, the results were analyzed using SPSS 20 . The significance level of all tests was considered $\mathrm{P}<0.05$.

Result: The results showed that the mean scores of the Pittsburgh sleep quality questionnaire at the beginning of general military training and the end of the training period were $8.5 \pm 2.6$ and $12.8 \pm 2.9$, respectively $(\mathrm{P}<0.05)$. The results showed that there was a statistically significant difference in all dimensions of the questionnaire except for a delay in sleep.

Discussion and Conclusion: Considering the decreased sleep quality., it is suggested to provide context for organizing group counseling sessions, reducing night wakes, improving nutrition, and sports activities.

Keyword: General Military Training, Military Service, Sleep Disturbance, Soldier. 


\section{تأثير دوره آموزش عمومى نظام وظيفه بر كيفيت خواب سربازان}

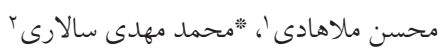

جكيده

مقدمه: افراد نظامى و به ويزه سربازان به دليل مأموريتهاى ويزه، مسائل شغلى، فشارهاى روانى بيش از اندازه، قوانين

سخت نظامى، نسبت به افراد غير نظامى در معرض آسيب بيشترى از جهت سلامت عمومى و اختلال خواب هستند.

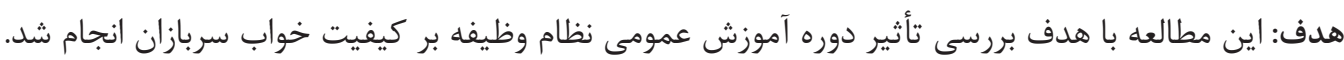

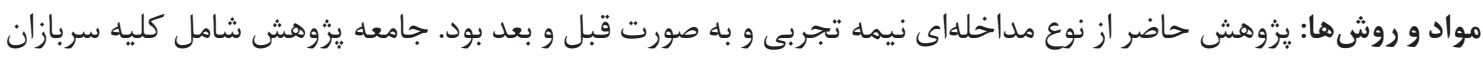

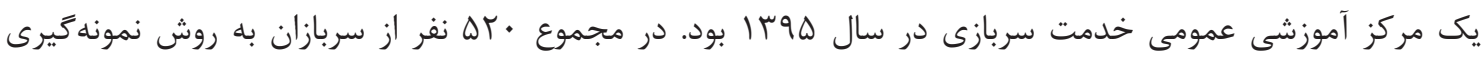

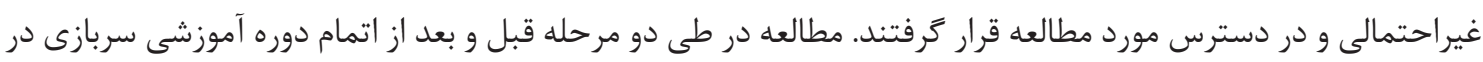

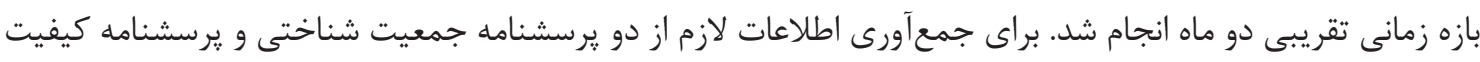

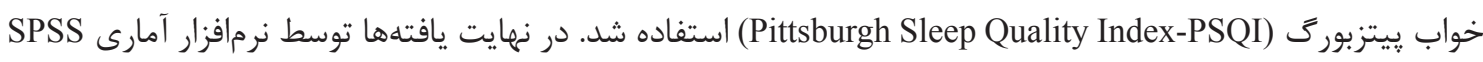

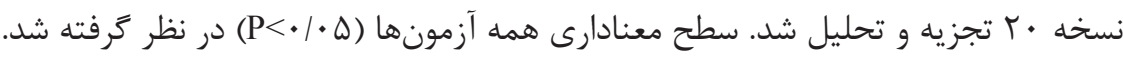

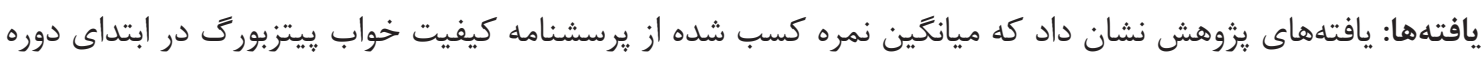

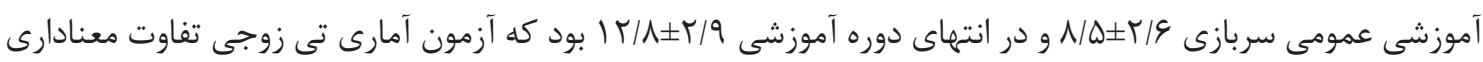

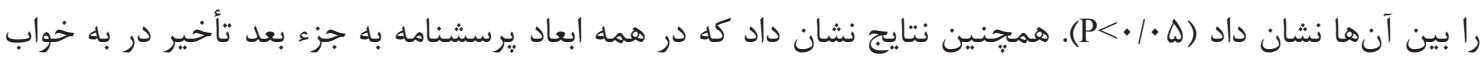

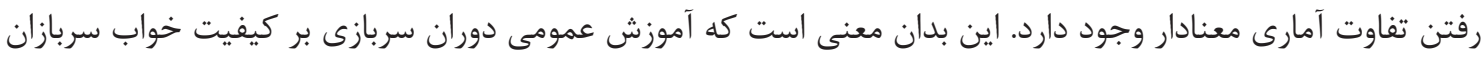
اثر مى كذارد.

بحث و نتيجه گيرى: با توجه به افت كيفيت خواب، بركزارى جلسات مشاوره گروهى، تقليل بيدارباشهاى شبانه، بهبود تغذيه و ايجاد بسترى مناسب براى انجام فعاليتهاى ورزشى ييشنهاد مى شود.

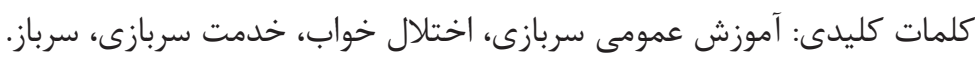

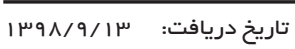

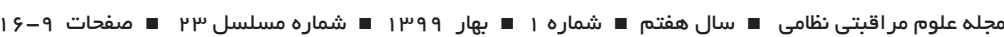
|تاريخ يذيرش: : تاريخ دريافت:

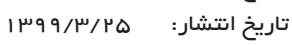

دنياى غرب اختلالات خواب را تجربه مى كنند و حدود ها تا •r مقلمهـ درصد اين افراد از اختلالات خواب مزمن رنج مى برند (باّ)، جنان در مبحث سلامت و رفاه عمومى ابعاد مختلفى از جمله: علائم كه اين آمار در مراكز مراقبتهاى اوليه بهداشتى (Primary Care)

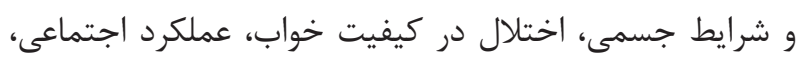
r Settings)

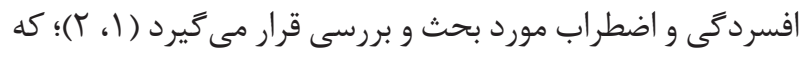
در ايالات متحده آمريكا •له تا • V ميليون نفر از انواع اختلالات خواب رنج مىبرند (ه). اختلالات خواب مى تواند در عملكرد روزانه

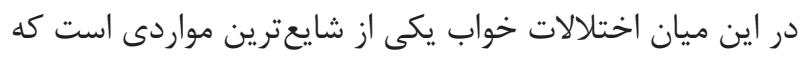

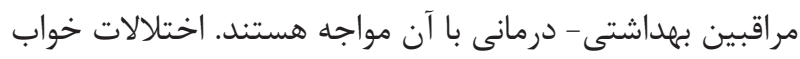

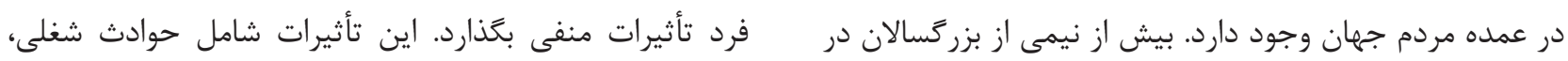


در يرسنل نظامى ايالات متحده كه اختلالات خواب دارند، بسيار

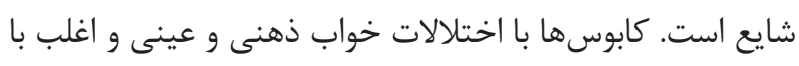
ساير اختلالات خواب و سلامت روانى همراه هستند (ه ().

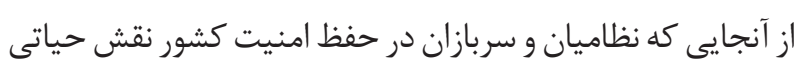

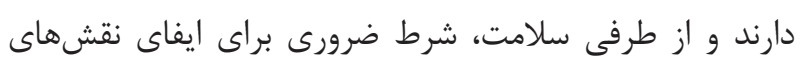

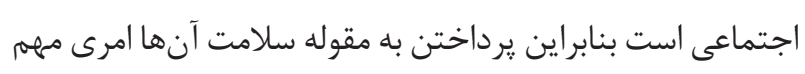

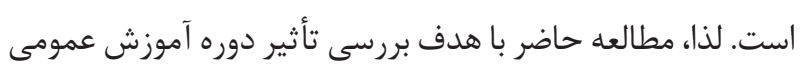
نظام وظيفه بر كيفيت خواب سربازان انجام شد.

\section{مواد و روشها}

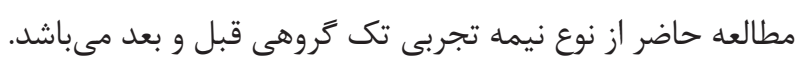

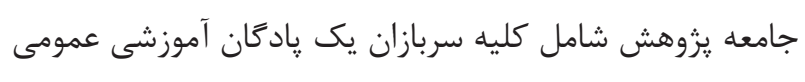

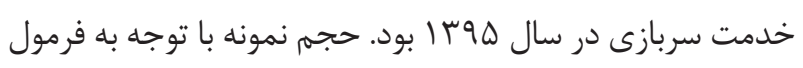
محاسبه حجم نمونه كوكران •له نفر كه با توجه به استقبال

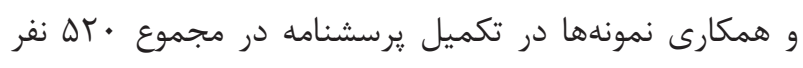

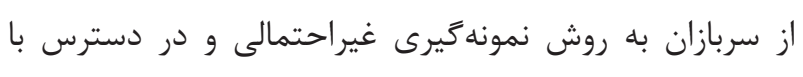

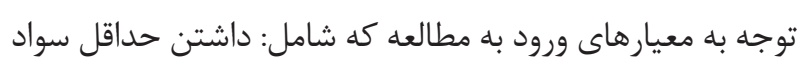

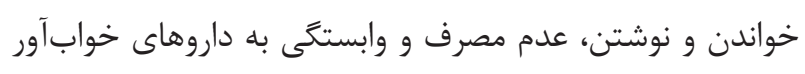

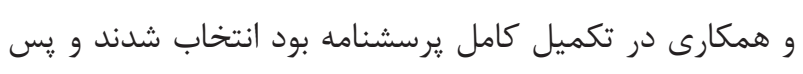

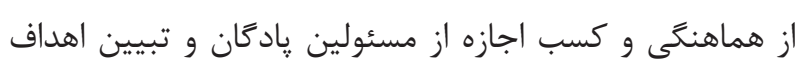
مطالعه، كسب رضايت آكاهانه از آنها وارد مطالعه شده و دمر مور مورد كمنام بودن، حفظ اسرار و رعايت حريم خصوصى آنان اطمينان

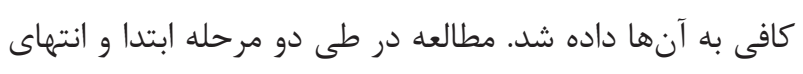

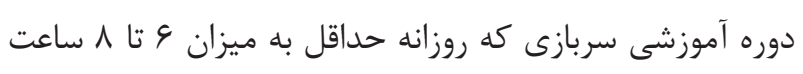

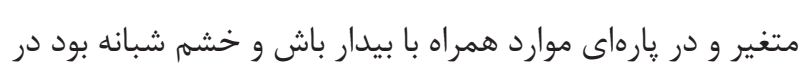

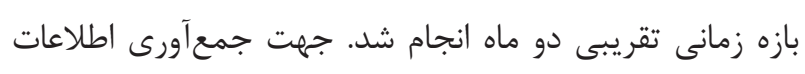

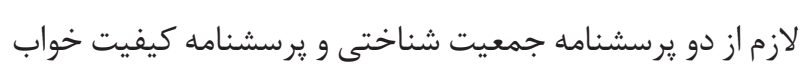
ييتزبورى (Pittsburgh Sleep Quality Index-PSQI) استفاده شد. يرسشنامه جمعيت شناختى شامل سؤالاتى از قبيل وضعيت

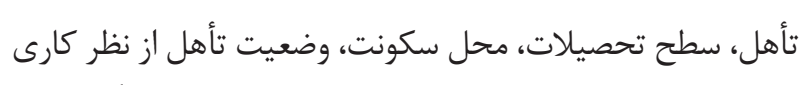

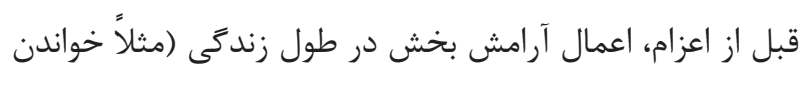

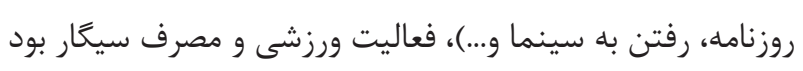
كه روايى آن به شيوه روايى محتوا توسط ده نفر از اعضاء هيئت علمى بررسى و تائيد گرديد.
رانندگى، مشكلات روانيزشكى و افت كيفيت زندَّى مىشود؛ كه در نهايت منجر به اختلال در سلامت عمومى خواهد شد (1-1).

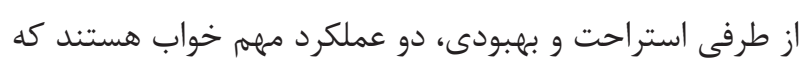

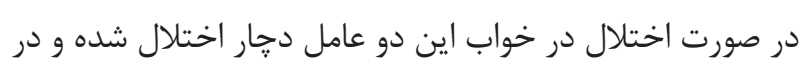

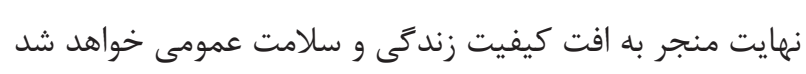
(9). اختلالات خواب منجر به افزايش مرى و مير، صدمات روحى نى ونى

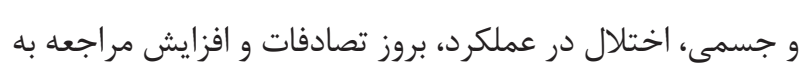
مراكز درمانى مىشود ( • (1).

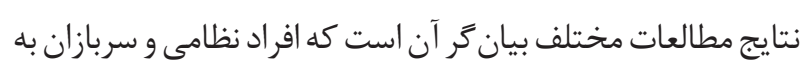
دليل ماهيت كارى از جمله سختى كار، مأموريتهاى ويزّه، مسائل

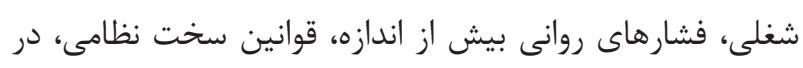
معرض آسيب بيشتر در سلامت عمومى و اختلالات خواب نسبت رواني به افراد غير نظامى هستند (1) (1). در مطالعه محمدى و همكاران

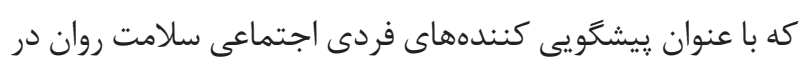

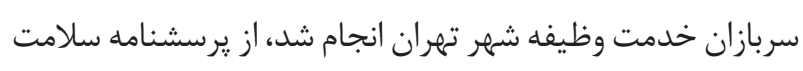
عمومى (GHQ) استفاده شد. نتايج نشان داد كه زير مقياس اختلال

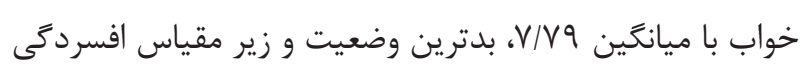

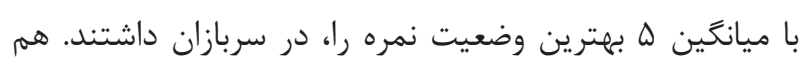
جنين در زير مقياسهاى يرسشنامه سلامت عمومى، بيشترين

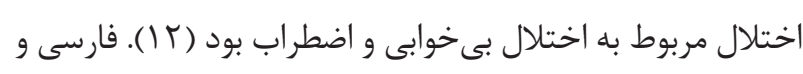

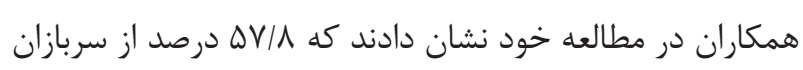

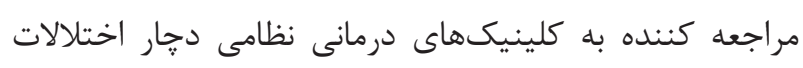

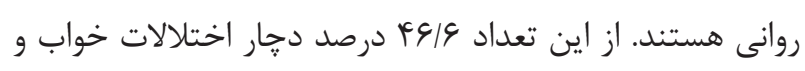

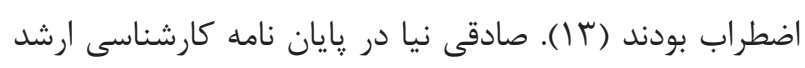

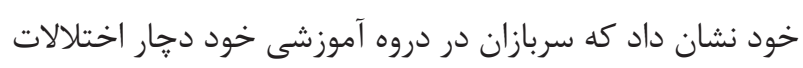

روانى و اختلالات خواب مىشوند (4 أ (1).

انجمن : يزشكى آمريكا Medical Surveillance Monthly Report)

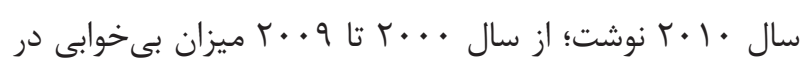

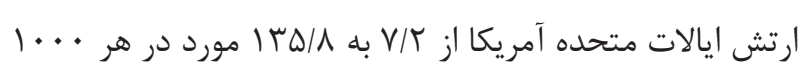

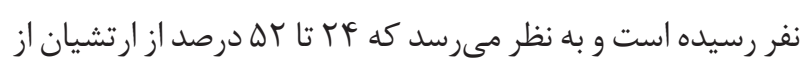
بى خوابى رنج مى برند ( • (). علاوه بر اين، مشكلات ديخرى هم همزمان

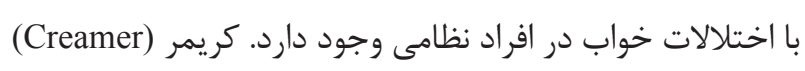
در مطالعه خود نشان داد كه كابوسهاى قابل توجه از نظر بالينى دابل 
مذهبى را سبب آرامش روحى و روانى، س/ آ درصد فعاليت ورزشى

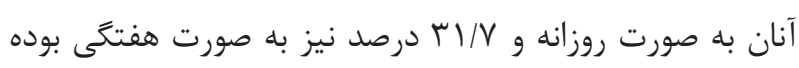

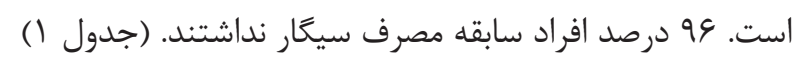

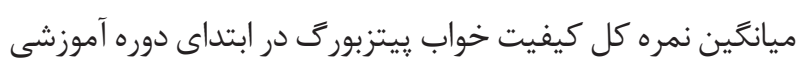

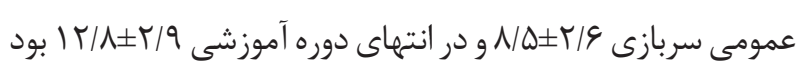
كه آزمون آمارى تى زوجى تفاوت معنادارى بين آنها نشان دادي

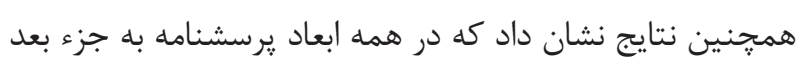

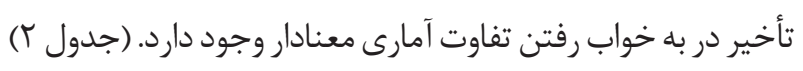
همجنين به منظور بررسى ارتباط برخى از مشخصات دموَر افيك

جدول ا- مشخصات دموكر افيك واحدهاى مورد يثوهش

\begin{tabular}{|c|c|c|c|}
\hline \multicolumn{3}{|c|}{ فراوانى } & \multirow{2}{*}{ متغير } \\
\hline درصد & & تعدا & \\
\hline $91 / \pi$ & FVF & مجرد & \multirow{3}{*}{ وضعيت تأهل } \\
\hline $4 / 9$ & TY & نامزد & \\
\hline$F / T$ & rT & متأهل & \\
\hline f & rI & ابتدايى & \multirow{4}{*}{ سطح تحصيلات } \\
\hline$f \cdot 19$ & 111 & سيكل & \\
\hline$F \Delta / \Lambda$ & TrA & دييلم & \\
\hline $9 / 9$ & $\Delta \cdot$ & فوق دييله & \\
\hline $1 \cdot / 4$ & $\Delta r$ & روستا & \multirow{3}{*}{ محل سكونت } \\
\hline$r 1 / 9$ & 114 & شهركوجى & \\
\hline $9 V / 9$ & rut & شهر بزرى & \\
\hline $\mid F / T$ & VF & محصل & \multirow{3}{*}{ وضعيت قبل از اعزام } \\
\hline $99 / \pi$ & MFY & شاغل & \\
\hline $19 / 9$ & $1 \cdot r$ & بيكار & \\
\hline FT/K & tr. & مراسم مذهبى & \multirow{6}{*}{ اعمال آرام بخش } \\
\hline$r T / \mu$ & 119 & ورزش & \\
\hline$r / V$ & 19 & مطالعه & \\
\hline$\Lambda / V$ & $i d$ & با دوستان & \\
\hline 10 & VA & موسيقى & \\
\hline $1 / 1$ & et & تفريح & \\
\hline 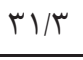 & 194 & روزانه & \multirow{4}{*}{ فعاليت ورزشى } \\
\hline$r I / V$ & 190 & هفتخى & \\
\hline TT/V & $1 V \cdot$ & موردى & \\
\hline$r / T$ & rt & اصلاً & \\
\hline 9 & 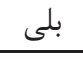 & بلى & \multirow{2}{*}{ مصرف سيعار } \\
\hline 94 & خير & خير & \\
\hline
\end{tabular}

يرسشنامه كيفيت خواب ييتزبورى (PSQI) يك يرسشنامه

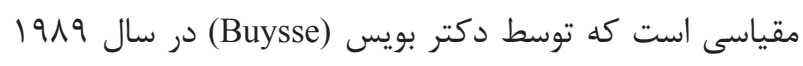

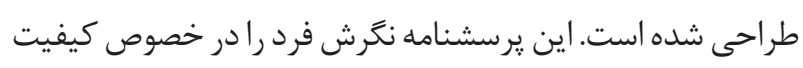

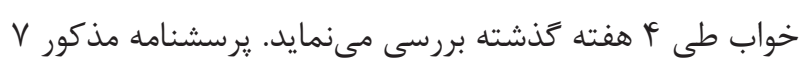

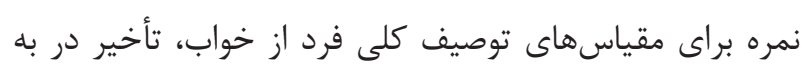

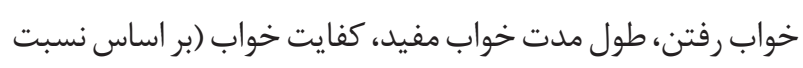

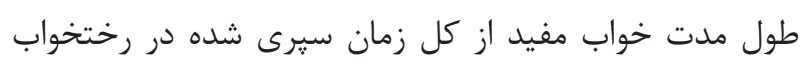

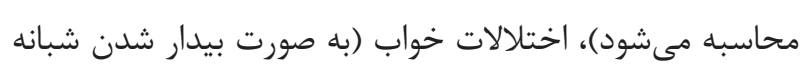

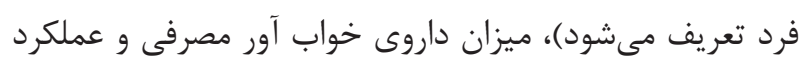
صبحكاهى (به صورت مشكلات تجربه شده توسط فرد در طول

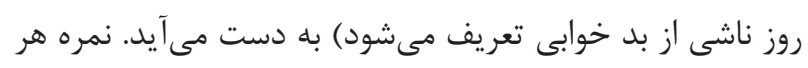

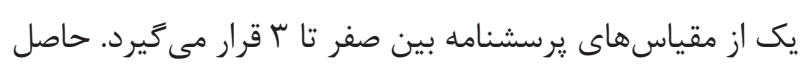

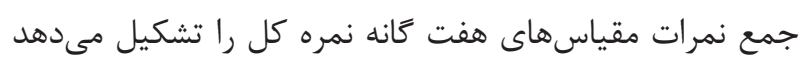

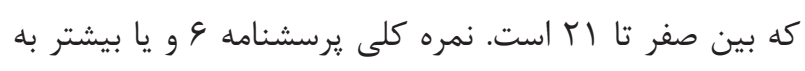

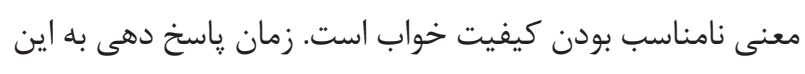

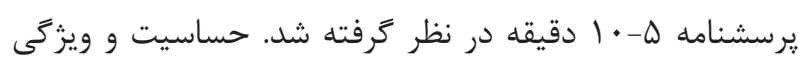

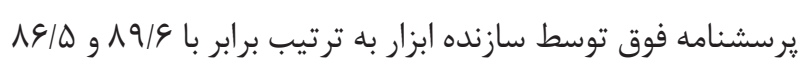
درصد كزارش شده است (9 (). اين ابزار به دفعات زياد در مطالعات

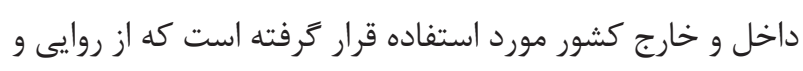

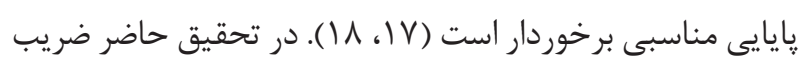

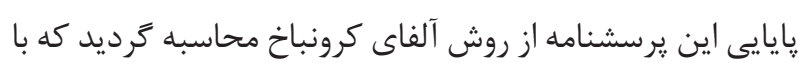

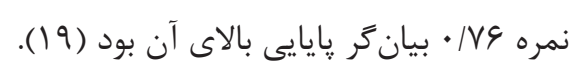

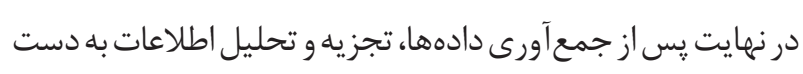

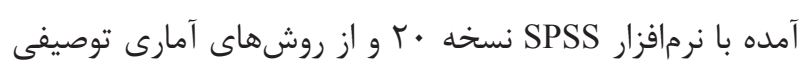

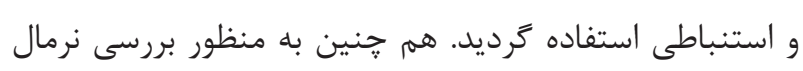
بودن توزيع دادها از آزمون كولموكروف-اسميرونوف استفاده شد اسند

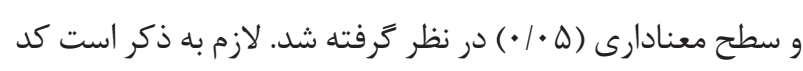

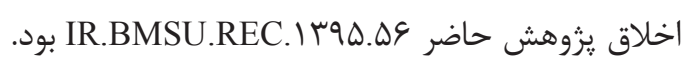

يافته ها اكثريت واحدهاى مورد يزوهش (T// (9 درصد) مجرد، در حدود

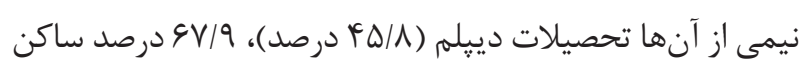

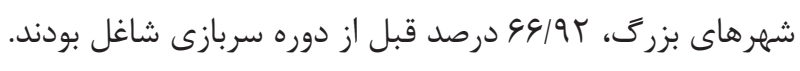

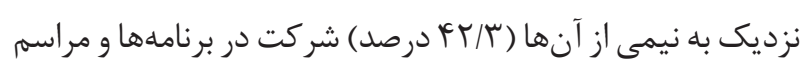


جدول r- اختلاف ميانكين نمره كيفيت خواب واحدهاى مورد يثرهش و ابعاد آن قبل و بعد از دوره آموزشى

\begin{tabular}{|c|c|c|c|c|}
\hline آمارى & & 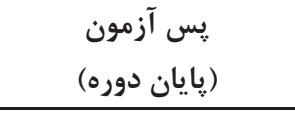 & ي شيش آزمون & \multirow{2}{*}{ ابعاد كيفيت خوره آموزشى } \\
\hline \multicolumn{2}{|c|}{ سطح معنادارى و آماره آزمون } & ميانگين (انحراف معيار) & ميانغين (انحراف معيار) & \\
\hline $\mathrm{P}=\cdot 1 \ldots *$ & $t=r / \Delta$ & $1 / \Lambda(\cdot / V)$ & $1 / 9(\cdot / V)$ & توصيف كلى فرد از خواب \\
\hline \multicolumn{2}{|c|}{$\begin{array}{c}\mathrm{P}=\cdot / \uparrow \Delta \\
\mathrm{t}=\cdot / \mathrm{V}\end{array}$} & $1 / \Lambda(\cdot / V)$ & $1 / \Lambda(\cdot / V)$ & تأخير در به خواب رفتن \\
\hline $\mathrm{P}=\cdot / \ldots *$ & $\mathrm{t}=\omega / \mathrm{\omega}$ & $1 / \Lambda(\cdot / V)$ & $1 / 9(\cdot 19)$ & طول مدت خواب مفيد \\
\hline $\mathrm{P}=\cdot / \cdots *$ & $\mathrm{t}=\Delta / \mathrm{r}$ & $1 / 9(\cdot 19)$ & $1 / \Lambda(\cdot / V)$ & كفايت خواب \\
\hline $\mathrm{P}=\cdot / \cdots *$ & $\mathrm{t}=r \varphi / 1$ & $1 / \wedge(\cdot / V)$ & $\cdot 10(\cdot / \mathrm{V})$ & اختلالات خواب \\
\hline $\mathrm{P}=\cdot / \cdots *$ & $\mathrm{t}=r \mathrm{r} / \mathrm{r}$ & $1 / 9(\cdot / \mathrm{V})$ & $.19(\cdot 19)$ & ميزان داروى خوابآور مصرفى \\
\hline $\mathrm{P}=\cdot / \cdots *$ & $\mathrm{t}=r q / r$ & $1 / V(\cdot 19)$ & $\cdot / \cdot \Lambda(\cdot / 4)$ & عملكرد صبحكاهى \\
\hline $\mathrm{P}=\bullet / \ldots *$ & $t=r q / V$ & $1 Y / \Lambda(Y / 9)$ & $N / D(T / 9)$ & حيطه كل \\
\hline
\end{tabular}

سربازان با كيفيت خواب آنان نتايج نشان داد كه بين وضعيت ق قبل از دوره آموزشى تفاوت و از نظر آمارى اختلاف معنادارى وجود

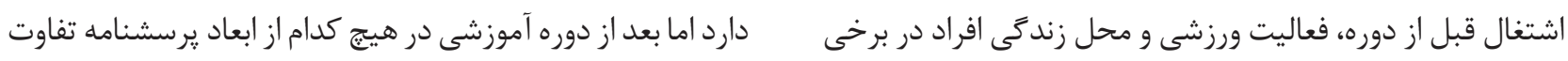

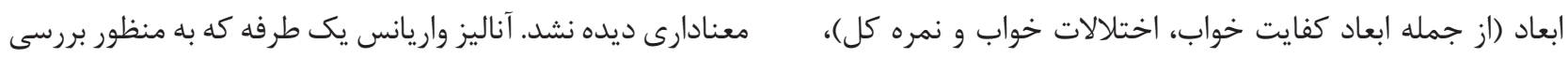
جدول ب - اختلاف ميانغين نمره كيفيت خواب و ابعاد آن با وضعيت اشتغال واحدهاى مورد يزوهش قبل و بعد از دوره آموزشى وضعيت اشتغال

\begin{tabular}{|c|c|c|c|c|c|c|c|c|}
\hline \multirow{2}{*}{ معنادارى } & \multicolumn{3}{|c|}{ وضعيت اشتغال بعد از دوره آموزشى } & \multirow{2}{*}{ معنادارى سطح } & \multicolumn{3}{|c|}{ وضعيت اشتغال قبل از دوره } & \multirow[t]{2}{*}{ ابعاد كيفيت خواب } \\
\hline & بيكار & شاغل & محصل & & بيكار & شاغل & محصل & \\
\hline $\begin{array}{l}\mathrm{F}=\cdot / \mathrm{VV} \\
\mathrm{P}=\cdot / \mathrm{V}\end{array}$ & $1 / \wedge(\cdot / V)$ & $1 / \wedge(\cdot / V)$ & $1 / \wedge(\cdot / V)$ & $\begin{array}{l}\mathrm{F}=\cdot / 4 D \\
\mathrm{P}=\cdot 19\end{array}$ & $1 / 9(\cdot / V)$ & $r(\cdot / V)$ & $1 / 9(\cdot / 9)$ & توصيف كلى فرد از خواب \\
\hline $\begin{array}{l}\mathrm{F}=1 / \Delta \Lambda \\
\mathrm{P}=\cdot / \mathrm{r}\end{array}$ & $r(\cdot / V)$ & $1 / 9(\cdot 19)$ & $1 / 9(\cdot / V)$ & $\begin{array}{l}\mathrm{F}=\cdot / 99 \\
\mathrm{P}=\cdot / \mathrm{r}\end{array}$ & $1 / 9(\cdot / V)$ & $1 / 1(\cdot / V)$ & $1 / V(\cdot 19)$ & تأخير در به خواب رفتن \\
\hline $\begin{array}{l}\mathrm{F}=\cdot / \mathrm{VV} \\
\mathrm{P}=\cdot / \mathbb{4}\end{array}$ & $1 / 9(\cdot / V)$ & $1 / A(\cdot / V)$ & $1 / V(\cdot / V)$ & $\begin{array}{l}\mathrm{F}=1 / \mathrm{VI} \\
\mathrm{P}=\cdot / 1\end{array}$ & $1 / 9(\cdot / 9)$ & $1 / 9(\cdot 19)$ & $1 / 0(\cdot 19)$ & طول مدت خواب مفيد \\
\hline $\begin{array}{l}\mathrm{F}=\cdot / \wedge \varphi \\
\mathrm{P}=\cdot / \uparrow\end{array}$ & $1 / 9(\cdot 19)$ & $1 / 9(\cdot 19)$ & $1 / 0(\cdot 19)$ & $\begin{array}{l}\mathrm{F}=\cdot / \Delta r \\
\mathrm{P}=\cdot / \Delta\end{array}$ & $1 / \wedge(\cdot / V)$ & $1 / \Lambda(\cdot / V)$ & $1 / V(\cdot / \Lambda)$ & 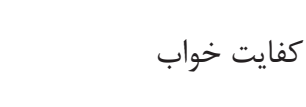 \\
\hline $\begin{array}{l}\mathrm{F}=1 / \mathrm{r} \\
\mathrm{P}=\cdot / \pi\end{array}$ & $1 / 9(\cdot / V)$ & $1 / A(\cdot / V)$ & $I / V(\cdot / V)$ & $\begin{array}{c}\mathrm{F}=\mathrm{F} / \mathrm{T} \\
\mathrm{P}=\cdot / \cdot 1 *\end{array}$ & $\cdot / \mathcal{F}(\cdot / V)$ & $\cdot / \Delta(\cdot \mid \Lambda)$ & $\cdot / r(\cdot / 9)$ & اختلالات خواب \\
\hline $\begin{array}{l}\mathrm{F}=\cdot / \Delta r \\
\mathrm{P}=\cdot / \Delta\end{array}$ & $r(\cdot / V)$ & $1 / 9(\cdot / V)$ & $1 / 9(\cdot / 1)$ & $\begin{array}{l}\mathrm{F}=r / \Delta \varphi \\
\mathrm{P}=\cdot / \cdot V\end{array}$ & $\cdot 19(\cdot / 1)$ & $.19(\cdot 19)$ & $\cdot / \mathbb{f}(\cdot \mid \Lambda)$ & مصيزان داروى خوابآور \\
\hline $\begin{array}{l}\mathrm{F}=\cdot / \wedge \varphi \\
\mathrm{P}=\cdot / 4\end{array}$ & $1 / 1(\cdot 19)$ & $1 / V(\cdot / 9)$ & $1 / 9(\cdot / 9)$ & $\begin{array}{l}\mathrm{F}=1 / \mathrm{VT} \\
\mathrm{P}=\cdot / 1\end{array}$ & $\cdot \mid \wedge(\cdot / \mathbb{f})$ & $\cdot / 1(\cdot / 4)$ & $\cdots(\cdot)$ & كرد صبحكَاهى \\
\hline $\begin{array}{l}\mathrm{F}=1 / \mathrm{Ar} \\
\mathrm{P}=\cdot / 1\end{array}$ & $\begin{array}{l}(r / 1) \\
1 K / 4\end{array}$ & $\begin{array}{l}(Y / \Lambda) \\
I T / \Lambda\end{array}$ & $\begin{array}{l}(r / 1) \\
1 T / 1\end{array}$ & $\begin{array}{c}\mathrm{F}=\Delta / \varepsilon \\
\mathrm{P}=\cdot / \cdots \kappa *\end{array}$ & $N / \Delta(T / V)$ & $\Lambda / V(T / 9)$ & $V / \varphi(T / T)$ & حيطه كل \\
\hline
\end{tabular}


خواب سربازان وظيفه انجام شد. نتايج مطالعه حاضر حاكى از آن

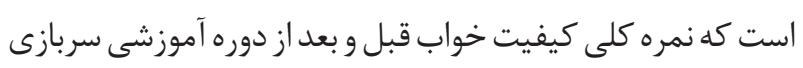

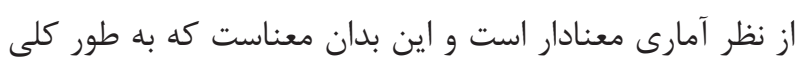

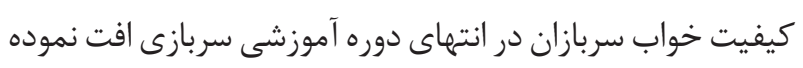

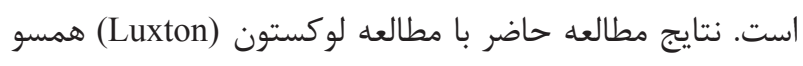

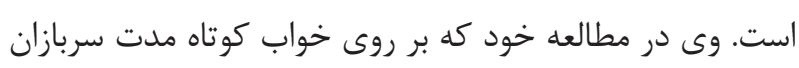

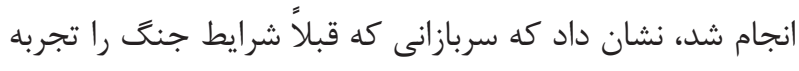
كردهاند بيشتر در معرض خطر اختلالات خواب مىشوند (·) (r). در مطالعه لوكستون بررسى سلامت و كيفيت خواب از جمله طول

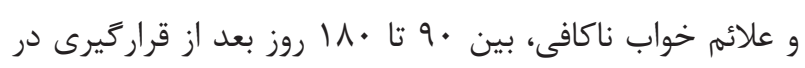

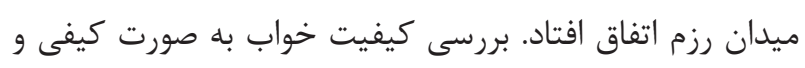

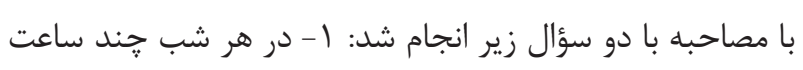

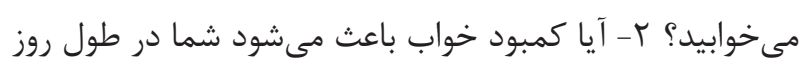

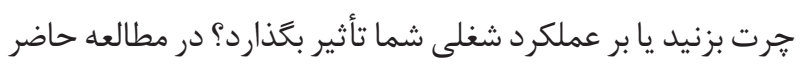

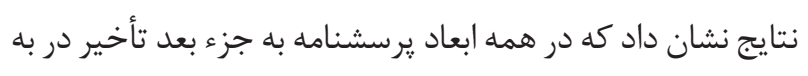

تفاوت معنادارى بين وضعيت اشتغال افراد قبل از دوره آموزشى

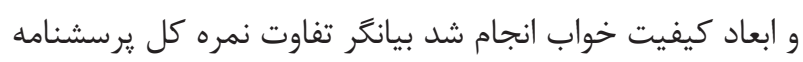
و بعد اختلالات خواب بين سه گروه محصل، شاغل و افراد بيكار

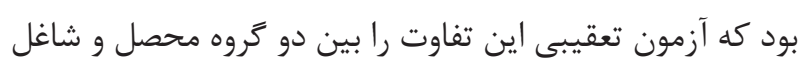

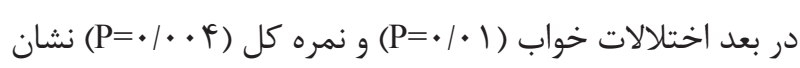

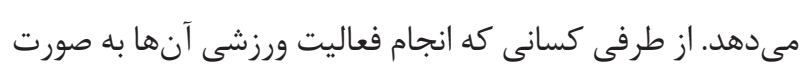
روزانه بود نمره بالاترى نسبت به سه كروه (هفتتى - كاهى و اصلاً)

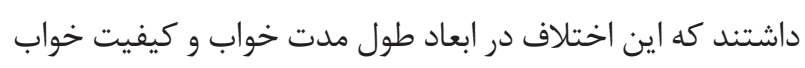

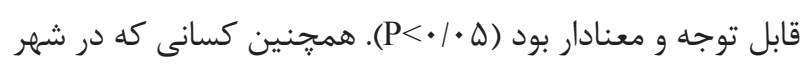

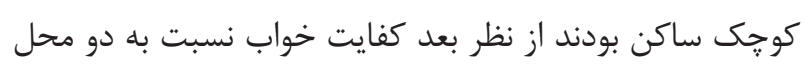

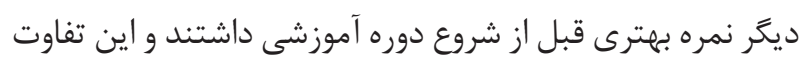

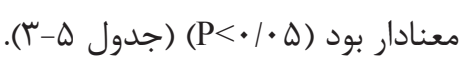

\section{بحث و نتيجهَكيرى}

مطالعه حاضر با هدف بررسى تأثير دوره آموزش سربيرى ني بر كيفيت

جدول F - اختلاف ميانغَين نمره كيفيت خواب و ابعاد آن با فعاليت ورزشى واحدهاى مورد يثوهش قبل و بعد از دوره آموزشى

\begin{tabular}{|c|c|c|c|c|c|c|c|c|c|c|}
\hline \multicolumn{10}{|c|}{ فعاليت ورزشى } & \multirow{3}{*}{ ابعاد كيفيت خواب } \\
\hline سطح & \multicolumn{4}{|c|}{ فعاليت وزشى قبل از دوره آموزشى } & \multirow{2}{*}{ سطادارى } & \multicolumn{4}{|c|}{ فعاليت ورزشى قبل از دوره آموزشى } & \\
\hline معنادارى & 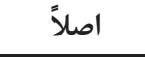 & كَاهى & هفتخَى & 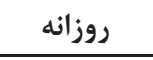 & & 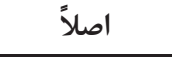 & كَاهى & هفتخَى & 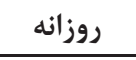 & \\
\hline $\begin{array}{l}\mathrm{F}=\cdot / \mu q \\
\mathrm{P}=\cdot / \mathrm{V}\end{array}$ & $1 / 9(\cdot / V)$ & $1 / \Lambda(\cdot / V)$ & $1 / \Lambda(\cdot / V)$ & $1 / \wedge(\cdot / 9)$ & $\begin{array}{l}\mathrm{F}=\cdot 1 \cdot 9 \\
\mathrm{P}=\cdot / 1\end{array}$ & $1 / \Lambda(\cdot / V)$ & $r(\cdot / V)$ & $1 / 9(\cdot / V)$ & $1 / 9(\cdot / 9)$ & توصيف كلى فرد \\
\hline $\begin{array}{l}\mathrm{F}=1 / \mathrm{k} \\
\mathrm{P}=\cdot / 4\end{array}$ & $r(\cdot / V)$ & $r(\cdot / V)$ & $1 / \Lambda(\cdot / V)$ & $r(\cdot / 9)$ & $\begin{array}{l}\mathrm{F}=\cdot 199 \\
\mathrm{P}=\cdot / 1\end{array}$ & $1 / \cdot 9(\mathrm{~V} / \cdot \mathrm{G})$ & $1 / 9(\cdot / V)$ & $1 / \Lambda(\cdot / V)$ & $1 / \wedge(\cdot / V)$ & تأخير در به خواب \\
\hline $\begin{array}{l}\mathrm{F}=1 / 4 q \\
\mathrm{P}=\cdot / r\end{array}$ & $1 / 9(\cdot / 1)$ & $1 / 9(\cdot / V)$ & $1 / V(\cdot / 9)$ & $1 / \Lambda(\cdot / V)$ & $\begin{array}{c}\mathrm{F}=r / \cdot r \\
\mathrm{P}=\cdot / \cdot r^{*}\end{array}$ & $1 / 9(\cdot / \Lambda)$ & $1 / 9(\cdot 19)$ & $1 / V(\cdot / 9)$ & $1 / 0(\cdot / 9)$ & مفيد مدل مدت خواب \\
\hline $\begin{array}{l}\mathrm{F}=\cdot / \mu \wedge \\
\mathrm{P}=\cdot / \mathrm{V}\end{array}$ & $1 / \Delta(\cdot / V)$ & $1 / 9(\cdot 19)$ & $1 / 9(\cdot / 9)$ & $1 / 0(\cdot / 9)$ & $\begin{array}{l}\mathrm{F}=\boldsymbol{r} / 9 . \\
\mathrm{P}=\cdot / \cdot r\end{array}$ & $T / /(\cdot / \Lambda)$ & $1 / 9(\cdot / 1)$ & $1 / \Lambda(\cdot / \Lambda)$ & $1 / 9(\cdot / \mathrm{V})$ & كفايت خواب \\
\hline $\begin{array}{l}\mathrm{F}=\cdot / \mathrm{V} \\
\mathrm{P}=\cdot / \Delta\end{array}$ & $1 / 9(\cdot / 1)$ & $1 / \Lambda(\cdot / \Lambda)$ & $1 / \Lambda(\cdot / V)$ & $I / V(\cdot / V)$ & $\begin{array}{c}\mathrm{F}=r / r \\
\mathrm{P}=\cdot / \cdot V\end{array}$ & $\cdot / 9(\cdot / 1)$ & $\cdot / \Delta(\cdot / V)$ & $\cdot / \mathbb{F}(\cdot \mid \Lambda)$ & $\cdot / \mathbb{f}(\cdot / \Lambda)$ & اختلالات خواب \\
\hline $\begin{array}{l}\mathrm{F}=1 / \mathrm{q} \\
\mathrm{P}=\cdot / \mathrm{r}\end{array}$ & $T / \mid(\cdot / \Lambda)$ & $1 / 9(\cdot / V)$ & $r(\cdot / \Lambda)$ & $1 / 9(\cdot / V)$ & $\begin{array}{l}F=r / r \Delta \\
P=\cdot 1 \cdot \Lambda\end{array}$ & $\cdot / 1(\cdot / 9)$ & $\cdot 19(\cdot 19)$ & $\cdot 19(1)$ & $\cdot 10(\cdot / 1)$ & خيزان داروى \\
\hline $\begin{array}{l}\mathrm{F}=1 / 1 . \\
\mathrm{P}=\cdot / \Gamma\end{array}$ & $1 / \Lambda(\cdot 19)$ & $1 / 1(\cdot 19)$ & $1 / V(\cdot / V)$ & $1 / 9(\cdot 19)$ & $\begin{array}{l}\mathrm{F}=\cdot / 9 \mathrm{~F} \\
\mathrm{P}=\cdot / \mathrm{f}^{\mathrm{F}}\end{array}$ & $\cdot / \cdot F(\cdot / T)$ & $\cdot / \cdot \Delta(\cdot / \mu)$ & $\cdot / 1(\cdot / \Delta)$ & $\cdot / V(\cdot / \mathbb{F})$ & عملكرد صبحكاهى \\
\hline $\begin{array}{l}\mathrm{F}=\cdot / 49 \\
\mathrm{P}=\cdot 19\end{array}$ & $\mid r / \cdot(r / \mathcal{F})$ & $|r /|(Y / \cdot q)$ & $\mid K / \Lambda(Y / \Lambda)$ & $I T / V(T / 9)$ & $\begin{array}{l}\mathrm{F}=r / \Delta r \\
\mathrm{P}=\cdot / \cdot 1 *\end{array}$ & $9 / \Delta(r / \Delta)$ & $\Lambda / \Lambda(\Gamma / Q)$ & $\wedge(Y / V)$ & $V(Y / \Delta)$ & حيطه كل \\
\hline
\end{tabular}


جدول ه - اختلاف ميانغَين نمره كيفيت خواب و ابعاد آن با محل زندكى واحدهاى مورد يثوهش قبل و بعد از دوره آموزشى

\begin{tabular}{|c|c|c|c|c|c|c|c|c|}
\hline \multicolumn{8}{|c|}{ محل سكونت } & \multirow{3}{*}{ ابعاد كيفيت خواب } \\
\hline \multirow{2}{*}{ 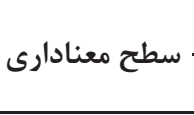 } & \multicolumn{3}{|c|}{ محل زندكى قبل از دوره آموزشى } & \multirow{2}{*}{ سطح معنادارى } & \multicolumn{3}{|c|}{ محل زندكى قبل از دوره آموزشى } & \\
\hline & شهر بزرى & شهر كوجى & ل - موستا & & شهر بزرى & شهر كوجى & روستا & \\
\hline $\begin{array}{l}\mathrm{F}=\cdot / 9 \mu \\
\mathrm{P}=\cdot / \Gamma\end{array}$ & $1 / \Lambda(\cdot / V)$ & $1 / V(\cdot / V)$ & $1 / 1(\cdot / 9)$ & $\begin{array}{l}\mathrm{F}=\cdot / \Delta \mathrm{V} \\
\mathrm{P}=\cdot / \Delta\end{array}$ & $r(\cdot / V)$ & $1 / 9(\cdot / 9)$ & $r(\cdot / V)$ & توصيف كلى فرد از \\
\hline $\begin{array}{l}\mathrm{F}=\cdot / \mu \omega \\
\mathrm{P}=\cdot / \varphi\end{array}$ & $r(\cdot / V)$ & $1 / 9(\cdot 19)$ & $r(\cdot / V)$ & $\begin{array}{l}\mathrm{F}=1 / \mathrm{V} . \\
\mathrm{P}=\cdot / 1\end{array}$ & $1 / \Lambda(\cdot / V)$ & $1 / V(\cdot 19)$ & $r(\cdot / V)$ & تأخير در به خواب رفتن \\
\hline $\begin{array}{l}\mathrm{F}=1 / 11 \\
\mathrm{P}=\cdot / /\end{array}$ & $r(\cdot / V)$ & $I / V(\cdot / V)$ & $1 / 9(\cdot / V)$ & $\begin{array}{l}\mathrm{F}=r / \mathcal{}(\mathrm{T} \\
\mathrm{P}=\cdot / \cdot \Lambda\end{array}$ & $1 / 9(\cdot 19)$ & $1 / 9(\cdot / 9)$ & $1 / 1(\cdot 19)$ & طول مدت خواب مفيد \\
\hline $\begin{array}{l}\mathrm{F}=\cdot / 4 q \\
\mathrm{P}=\cdot 19\end{array}$ & $1 / 9(\cdot 19)$ & $1 / \Delta(\cdot / V)$ & $1 / 9(\cdot 19)$ & $\begin{array}{c}\mathrm{F}=r / \Delta \mathrm{V} \\
\mathrm{P}=\cdot / \cdot r^{*}\end{array}$ & $1 / \wedge(\cdot / V)$ & $1 / 9(\cdot 19)$ & $1 / 9(\cdot / \mathrm{V})$ & كفايت خواب \\
\hline $\begin{array}{l}\mathrm{F}=1 / T r \\
\mathrm{P}=\cdot / T\end{array}$ & I/A $(\cdot / \mathrm{V})$ & $I / V(\cdot / \Lambda)$ & $1 / 1(\cdot / 9)$ & $\begin{array}{l}\mathrm{F}=\cdot 1 \cdot 9 \\
\mathrm{P}=\cdot 19\end{array}$ & $\cdot / \Delta(\cdot / V)$ & $\cdot / \Delta(\cdot \mid \Lambda)$ & $\cdot / 0(\cdot / V)$ & اختلالات خواب \\
\hline $\begin{array}{l}\mathrm{F}=1 / \mathrm{rq} \\
\mathrm{P}=\cdot / 4\end{array}$ & $1 / 9(\cdot / V)$ & $1 / 9(\cdot / \mathrm{V})$ & $r / /(\cdot / \Lambda)$ & $\begin{array}{c}\mathrm{F}=\cdot / \mathrm{\omega} \\
\mathrm{P}=\cdot / \mathrm{V}\end{array}$ & $\cdot 19(\cdot 19)$ & $.19(\cdot / 1)$ & $\cdot 19(\cdot / 9)$ & مصيزان داروى خوابآور \\
\hline $\begin{array}{l}\mathrm{F}=\cdot 19 \mathrm{~V} \\
\mathrm{P}=\cdot / 0\end{array}$ & $1 / V(\cdot / 8)$ & $1 / V(\cdot / 8)$ & $1 / V(\cdot 19)$ & $\begin{array}{l}\mathrm{F}=r / \Delta \varphi \\
\mathrm{P}=\cdot / \cdot V\end{array}$ & $\cdot 19(\cdot / r)$ & $\cdot / 1(\cdot / 9)$ & $\cdot / \cdot 1(\cdot / 1)$ & 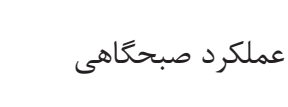 \\
\hline $\begin{array}{l}F=r / T F \\
P=\cdot / 1\end{array}$ & $1 T / 9(T / 9)$ & $\mid T / F(T / 1)$ & $\mid r / r(r / 1)$ & $\begin{array}{l}\mathrm{F}=\cdot / \wedge r \\
\mathrm{P}=\cdot / \boldsymbol{F}\end{array}$ & $N / \Delta(Y / \varphi)$ & $\Lambda / \mathcal{F}(Y / \Lambda)$ & $N / 9(T / 1)$ & حيطه كل \\
\hline
\end{tabular}

مشكلات خواب در نيروهاى نظامى رسمى و در فاصله زمانى حدود • ا ساله بررسى شده است. در حالى كه مطالعه حاضر در دورهاى كوتاه و بر روى سربازان وظيفه انجام شده است. هم جنين فرهنَ نيروهاى نظامى و شرايط آنها در دو كشور ايران و آمريكا متفاوت است كه اين مسئله مىتواند موجب اختلافاتى در نتايج دو مطالعه شود. ميسلوويج و همكاران (Mysliwiec\&etal) در مطالعه خود به بررسى اختلالات خواب در نيروهاى نظامى ايالات متحده يرداختند. وى بعد از 11 ماه از بازخشت نيروهاى نظامى از عمليات نظامى بـى

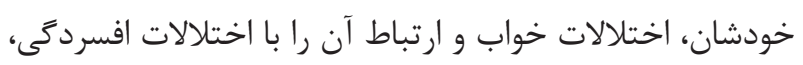
وجود درد در آنها و اختلالات استرس يس از سانحه بررسى كردند. به طور كلى، ع/V درصد معيارهاى تشخيصى براى آينه انسدادى حين خواب و و/ /ع درصد بىخوابى داشتند. هم جنين اين افراد در معرض خطر براى ابتلا به افسردگى و اختلال استرس يس از سانحه بودند (Tr). مطالعه ميسلوويج از نظر بروز اختلالات خواب بعد از دوره نظامى با مطالعه حاضر همسو است. در مطالعه
خواب رفتن تفاوت آمارى معنادار وجود دارد؛ كه با نتايج مطالعه فوق همخوانى و اختلال خواب را در سربازان نشان مىدهد. در مطالعه صادقى يور و همكاران كه با عنوان بررسى كيفيت زندكى و كيفيت خواب سربازان فعال و غيرفعال، · ·. سرباز با روش نمونهگيرى در دسترس انتخاب و بر اساس ميزان فعاليت ورزشى به دو گروه فعال و غيرفعال تقسيم شدند. سيس ميزان كيفيت زندگى و شرايط خواب آنها با استفاده از يرسشنامه كالدول و همكاران (Caldwell\&etal) در مطالعه خود به بررسى بى خوابى

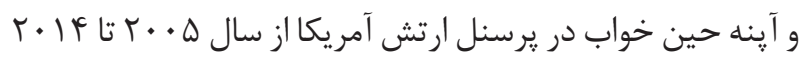
يرداختند. نتايج مطالعه آنها نشان داد كه مشكلات مربوط به

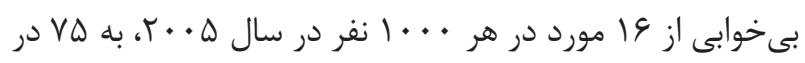
سال f| أ ب افزايش يافته است. هم جنين اين مشكلات در افراد سياه يوست، زنان و افسران ارشد، به نسبت ساير يرسنل نظامى،

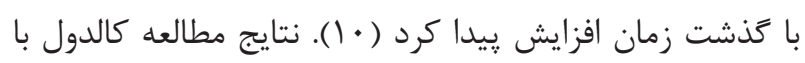
مطالعه حاضر از جهت افزايش مشكلات خواب با كذشت زمان و حضور در محيط نظامى همسو است. البته در مطالعه كالدول 
و اضطراب همراه بوده است. با توجه به لزوم آمادگى جسمى و روحى نيروهاى نظامى، لزوم برنامهريزى و ايجاد شرايط بهتر

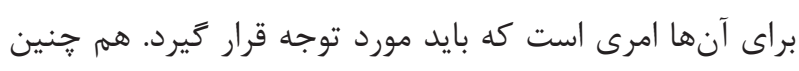
مىتوان با برنامهريزى در زمينه افزايش كيفيت وضعيت روحى إنى روانى مانند برگزارى جلسات مشاوره، زمينه سازى براى انجام ورزش در نيروهاى نظامى، بهبود تغذيه تا حدودى از مشكلات

$$
\text { ناشى از افت كيفيت خواب كاست. }
$$

از محدوديتهاى يزوهش حاضر مىتوان به تك گروهى بودن

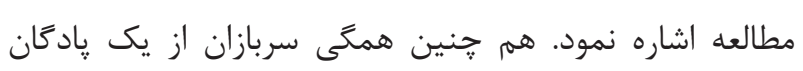

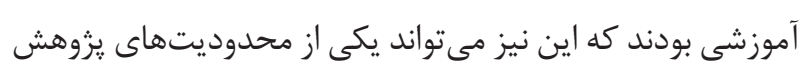
حاضر باشد و تعميم پِيرى را كاهش دهد. با توجه به محدوديتهاى ״رُوهش حاضر يِيشنهاد مى شود محققين

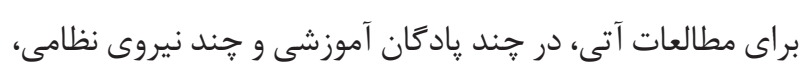
كيفيت خواب را بررسى نمايند.

\section{تقدير و تشكر}

از كليه فرماندهان و مسئولين پادگًان، همجنين از كليه سربازان معزز و كرامى كه در انجام اين يزوهش ما را يارى كردند كمال تشكر را داريم.

تضاد منافع

بدين وسيله نويسندگان تصريح مىنمايند كه هيج گونه تضاد منافعى در خصوص يزوهش حاضر وجود ندارد.

\section{References}

1- Hjelle EG, Bragstad LK, Zucknick M, Kirkevold M, Thommessen B, Sveen U. The General Health Questionnaire-28 (GHQ-28) as an outcome measurement in a randomized controlled trial in a Norwegian stroke population. BMC Psychol. 2019;7(1):18. https://doi.org/10.1186/s40359-019-0293-0

2- El-Metwally A, Javed S, Razzak HA, Aldossari KK, Aldiab A, Al-Ghamdi SH, et al. The factor structure of the general health questionnaire (GHQ12) in Saudi Arabia. BMC Health Serv Res. 2018;18 (1): 595. https://doi.org/10.1186/s12913-018-3381-6
ميسلوويج ارزشيابى خواب بر روى |f|4 | نيروى نظامى انجام شده

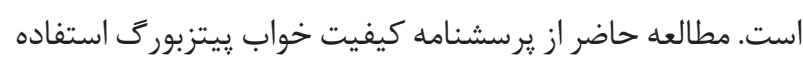

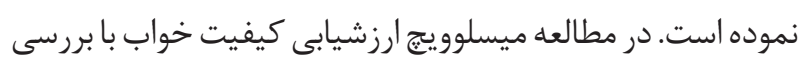
مواردى مانند يالس اكسيمترى، نوار مغزى، نوار قلب، نوار عضله

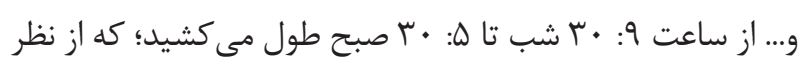
ابعاد بررسى با مطالعه حاضر متفاوت است. دانكر هوف و همكاران (Heidi Danker-Hopfe\&etal) با استفاده از مقياس سنجش خواب ييتزبرى كيفيت خواب 11 ال سرباز را كه در طول ع ماه در جنَ افغانستان شركت كرده بودند، قبل، حين

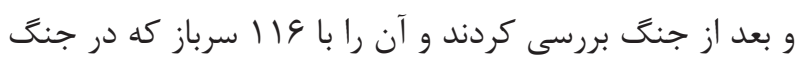
شر كت نكرده بودند، به عنوان زروه كنترل مقايسه كردند. در زروه

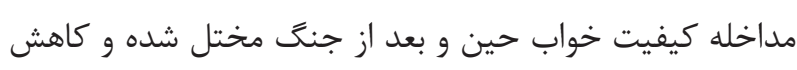
يافته بود (سץ). از بابت نتايج كلى دو مطالعه با يكديگر شباهت داشته و همسو بودند. البته در مطالعه دانكر هوف و همكاران بررسى در شرايط واقعى جنَ (Heidi Danker-Hopfe \& etal) صورت گرفته است، در حالى كه در مطالعه حاضر بعد از دوره آموزشى سربازى كه شرايط واقعى جنَ نيست مقايسه انجام شده

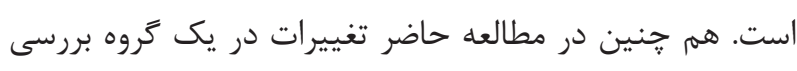
شده است، در حالى كه در مطالعه دانكر اين بررسى در دو گروه صورت گرفته است. نتايج به دست آمده از مطالعه حاضر و مطالعات مشابه، نشان از كاهش و افت كيفيت خواب در سربازان و نيروهاى نظامى در شرايط عادى و جنَ واقعى دارد. هم :هنين در برخى از مطالعات، افت كيفيت خواب، با افت كيفيت وضعيت روانى و ايجاد استرس

tool for sleep dysfunction in clinical and non-clinical samples: A systematic review and meta-analysis. Sleep medicine reviews. 2016;25:52-73. https://doi.org/10.1016/j.smrv.2015.01.009

4- Williams SG, Collen J, Wickwire E, Lettieri CJ, Mysliwiec V. The impact of sleep on soldier performance. Curr Psychiatr Rep. 2014;16(8):459. https://doi.org/10.1007/s11920-014-0459-7

5- Shin JC, Kim J, Grigsby-Toussaint D. Mobile phone interventions for sleep disorders and sleep quality: Systematic Review. JMIR mHealth and uHealth. 2017;5(9):e131. DOI: 10.2196/ mhealth. 7244

6- Fortier-Brochu E, Beaulieu-Bonneau S, Ivers H, Morin CM. 
Insomnia and daytime cognitive performance: A meta-analysis. Sleep Med Rev. 2012;16(1):83-94. https://doi.org/10.1016/j. smrv.2011.03.008

7- Guallar-Castillon P, Bayan-Bravo A, Leon-Munoz LM, BalboaCastillo T, Lopez-Garcia E, Gutierrez-Fisac JL, et al. The association of major patterns of physical activity, sedentary behavior and sleep with health-related quality of life: A cohort study. J Prev Med. 2014;67:248-54. https://doi.org/10.1016/j. ypmed.2014.08.015

8- Shekleton JA, Flynn-Evans EE, Miller B, Epstein LJ, Kirsch D, Brogna LA, et al. Neurobehavioral performance impairment in insomnia: relationships with self-reported sleep and daytime functioning. Sleep. 2014;37(1):107-16. https://doi.org/10.5665/ sleep. 3318

9- Fernández-Cruz KA, Jiménez-Correa U, Marín-Agudelo HA, Castro-López C, Poblano A. Proposing the Clinical Inventory of Sleep Quality. Sleep Sci. 2016;9(3):216-20. https://doi. org/10.1016/j.slsci.2016.10.002

10- J ACaldwell, Knapik JJ, Lieberman HR. Trends and factors associated with insomnia and sleep apnea in all United States military service members from 2005 to 2014. J Sleep RES. 2017;26(5):665-70. https://doi.org/10.1111/jsr.12543

11- Maleki B, Sanei S, Borhani H, A. G. Effect of military training on personality traits of military students. J Mil Med. 2012;3(4):195200. (Persian)

12- Mirghafourvand M, Mohammadi A, Effati Daryani F. Khavoshi N. Zarei S. Sociodemographic Predictors of Mental Health in Tehran's Soldiers 2012-2013. Journal of Military Medicine. 2015;17(2):81-7. (Persian)

13- Farsi Z, Jabari Morouei M, Ebadi A. General health assessment of Army soldiers seen in a military medical outpatient clinic in Tehran. Ann mil health sci Res. 2006;4(3):923-30. (Persian)

14- Sadeghinia A. The effect of a training course on some of the elements and physical aspects and mental health of soldiers in Ground Force garrison in sepah. tehran: Emam hosein university; 2006.
15- Creamer JL, Brock MS, Matsangas P, Motamedi V, Mysliwiec V. Nightmares in United States Military Personnel With Sleep Disturbances. J clin sleep Med. 2018;14(3):419-426. https://doi. org/10.5664/jesm.6990

16- Buysse DJ, Reynolds CF, Monk TH, Berman SR, Kupfer DJ. The Pittsburgh Sleep Quality Index: A new instrument for psychiatric practice and research. Psychiatry Res J. 1989;28(2):193-213.

17- Ehteshamzadeh P, marashi M. The relation between the intensity of insomnia, sleep quality sleepiness and mind mental health with educational operation in ahwaz male teenager. J soc psychol (new Findings in Psychology). 2010;5(15):27-38

18- Mehdizadeh S, Salaree M, Ebadi A, Aslani J, Naderi Z, Jafari Varjoshani N. Effect of Using Continuous Care Model on Sleep Quality of Chemical Warfare Victims with Bronchiolitis Obliterans. Hayat. 2010; 16 (2):5-14 (Persian)

19- Taber K. The use of Cronbach's alpha when developing and reporting research instruments in science education. Research in Science Education. 2018;48(6):1273-96. https://doi.org/10.1007/ s11165-016-9602-2

20- Luxton DD, Greenburg D, Ryan J, Niven A, Wheeler G, Mysliwiec V. Prevalence and Impact of Short Sleep Duration in Redeployed OIF Soldiers. Sleep. 2011;34(9):1189-95. https://doi.org/10.5665/ SLEEP.1236

21- Zar A, Rezaee F, Sadeghipour HR. The Survey of Life and Sleep Quality of Active and Inactive Soldiers. J Health. 2019;10(3):35968. (Persian)

22- Mysliwiec V, Gill J, Lee H, Baxter T, Pierce R, Barr TL, et al. Sleep disorders in US military personnel: A high rate of comorbid insomnia and obstructive sleep apnea. Chest. 2013;144(2):549-57. https://doi.org/10.1378/chest.13-0088

23- Danker-Hopfe H, Sauter C, Kowalski JT, Kropp S, Ströhle A, Wesemann U, et al. Sleep quality of German soldiers before, during and after deployment in Afghanistan - a prospective study. J sleep Res. 2017;26(3):353-63. https://doi.org/10.1111/jsr.12522 